\author{
PRELIMINARY RESULTS FROM E756 ON THE \\ $\Xi^{-}$AND $\Omega^{-}$MAGNETIC MOMENTS \\ K. Johns, J. Duryea, G. Guglielmo, K. Heller, M. Shupe*, K. Thorne \\ Physics Department, University of Minnesota, \\ Minneapolis, Mn. 55455 \\ C. James, K.B. Luk, R. Rameika \\ Fermilab, Batavia, Il. 60510 \\ P.M. Ho, M. Longo \\ Department of Physics, University of Michigan, Ann Arbor, Mi. 48109 \\ T. Diehl, S. Teige, G. Thomson \\ Physics Department, Rutgers - The State University, \\ Piscataway, NJ. 08854
}

\begin{abstract}
We have used the spin precession technique to measure the $\Xi^{-}$and $\Omega^{-}$ magnetic moments. The preliminary results are $\mu\left(\Xi^{-}\right)=-0.64 \pm 0.02$ nuclear magnetons and $\mu\left(\Omega^{-}\right)=-2.0 \pm 0.2$ nuclear magnetons where the error for both measurements is statistical. The polarization of $\Xi^{-1}$ s produced at $2.5 \mathrm{mr}$ by $800 \mathrm{GeV}$ protons on a Be target was $11 \%$ while the polarization of $\Omega^{- \text {'s }}$ was consistent with zero. Polarized $\Xi^{- \text {'s }}$ and $\Omega^{->}$s were produced using spin transfer from a polarized neutral hyperon beam. The $\Omega^{-}$polarization at $325 \mathrm{GeV} / \mathrm{c}$ was $6.5 \%$.
\end{abstract}

\title{
INTRODUCTION
}

The magnetic moments of atoms and particles has a distinguished history in helping us to understand the nature of interactions. This conference is devoted to spin so I need not remind you that spin was discovered by Stern and Gerlach while measuring the magnetic dipole moments of atoms. In this tradition today we believe the baryon magnetic moments can play an important role in the quest to understand quark confinement.

In the framework of the quark model, we can use the SU(6) wavefunctions to predict the baryon magnetic moments. They are just given as the expectation value of the magnetic moment operator

$$
\mu(\Lambda)=\sum_{i}<\Lambda \uparrow\left|\mu_{i} \sigma_{3 i}\right| \Lambda \uparrow>
$$

* Now at the University of Arizona 
Evaluating this expectation value gives, for example,

$$
\begin{aligned}
\mu(\Lambda)=\mu(s) & \mu(\Omega)=3 \mu(s) \\
\mu(p)=\frac{1}{3}(4 \mu(u)-\mu(d)) & \mu(n)=\frac{1}{3}(4 \mu(d)-\mu(u))
\end{aligned}
$$

Assuming $m(u)=m(d)$, the quark model predicts $\mu(n) / \mu(p)=-2 / 3$. Experimentally this ratio is found to be -0.68 which is in remarkable agreement. The $\Sigma$ and $\Xi$ moments are written like those for the proton or neutron but with the appropriate interchange of $u \rightarrow s$ or $d \rightarrow s$.

\begin{tabular}{|c|c|c|}
\hline & expermens & broken SU(6) \\
\hline$p$ & 2.794 & inpul \\
\hline$n$ & -1.913 & input \\
\hline$\Lambda$ & $-0.613 \pm 0.005$ & inpul \\
\hline$\Sigma^{*}$ & $2.38 \pm 0.02$ & 2.67 \\
\hline$\Sigma^{0}$ & $?$ & 0.79 \\
\hline$\Sigma^{\circ}$ & $-1.166 \pm 0.017$ & -1.09 \\
\hline$\Sigma \rightarrow \Lambda$ & $-1.59 \pm 0.09$ & -1.63 \\
\hline$\Xi^{\prime}$ & $-1.250 \pm 0.014$ & -1.44 \\
\hline$\Xi^{-}$ & $-0.69 \pm 0.04$ & -0.49 \\
\hline$\Omega^{-}$ & & -1.84 \\
\hline
\end{tabular}

TABLE 1. Experimental values and quark model predictions.

By using $\mu(p)$ and $\mu(n)$ to determine $\mu(u)$ and $\mu(d)$ and $\mu(\Lambda)$ to determine $\mu(\mathrm{s})$ we can predict the remainder of the baryon octet and $\Omega^{-}$moments. In Table 1 the quark model predictions are listed along with the experimentally measured values and associated error. There is fair agreement; the quark model gives the moments to within $10 \%$ of the experimental data. However the differences are significant given the 1-2\% errors of the measured values. It is reasonable to ask then whether these differences can be understood in the framework of the quark model.

To this end, a number of models have evolved which are additions or corrections to the basic quark model. These more sophisticated models include configuration mixing ${ }^{1,2}$, relativistic corrections ${ }^{3}$, and a quark mass or quark charge which depends on its baryon environment4,5. Alternative approaches to understanding baryon magnetic moments are provided by QCD sum rule6, bag model7,8 and QCD lattice calculations ${ }^{9}$.

In Figure 1, a large number of relevant predictions for the hyperon magnetic moments are plotted as the difference between theoretical prediction and

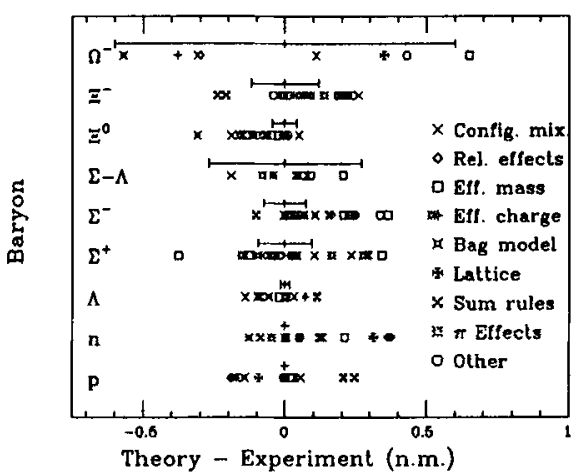

FIG. 1. Recent predictions for the baryon magnetic moments minus the experimentally measured values. experimental data. Above the predictions are the $3 \sigma$ error bars associated with the experimentally measured values. The details of which model is plotted where is not important here; the main purpose is to show the spread of the various calculations in relation to the precision of the experimental data. No model correctly predicts (within the 30 error bar) all the moments and most models correctly give only about half the moments they make predictions for.

One expects the $\Omega^{-}$to be an excellent system to distinguish among the various models. Its simple structure of 3 relatively heavy, identical, spin 
aligned quarks should make the $\Omega^{-}$more easily calculable. Furthermore, the $\Omega^{-}$ magnetic moment should give the most direct measurement of the strange quark moment. Thus the measurement of the $\Omega^{-}$magnetic moment will be an important aid to theorists.

\section{CALCULATION OF THE MAGNETIC MOMENT}

In $\mathrm{E} 756$, the $\Xi^{-}$and $\Omega^{-}$magnetic moments were determined by measuring their spin precession in a uniform magnetic field. The magnetic moment for charged particles is given by

$$
\bar{\mu}=\frac{g}{2} \frac{q}{m} \bar{S}
$$

where $\mathrm{g} / 2$ is the deviation from its Dirac moment. The torque on the dipole moment of a polarized hyperon in a uniform magnetic field gives rise to a precession angle measured in the lab system as

$$
\Phi_{l a b}=\frac{-q}{m \beta}\left(\frac{g}{2}-1+\frac{1}{\gamma}\right) \int B d l
$$

The momentum dependence arises as part of the Thomas precession contribution and may be eliminated by measuring the initial and final spin direetions with respect to the hyperon's momentum vector.

Since parity is conserved in the inclusive production of hyperons the initial spin direction is known and is given by

$$
\hat{n}=\left(\bar{k}_{p} \times \bar{k}_{\Omega}\right) /\left|\bar{k}_{p} \times \bar{k}_{\Omega}\right|
$$

Hence the net precession angle is found as

$$
\Phi_{n e t}=\tan ^{-1}\left(\frac{P_{z}}{P_{x}}\right)
$$

Since the decays $\Omega^{-} \rightarrow \Lambda K^{-}$and $\Xi^{-} \rightarrow \Lambda \Pi^{-}$are weak decays, the spin direction of the daughter $\Lambda$ is related to the spin direction of the parent via

$$
\bar{P}_{\Lambda}=\frac{1}{2(J+1)}\left[1+(2 J+1) \gamma_{\Omega}\right] \bar{P}_{\Omega}
$$

The problem of measuring the final spin direction after precession of $\Xi^{-}$'s or $\Omega^{- \text {'s }}$ is thus reduced to measuring the polarization of the daughter $\Lambda$ 's, for which a well known technique exists.

One measures the $\Lambda$ polarization by looking for an asymmetry in the angular distribution of the daughter proton in the $\Lambda$ rest frame. Because parity is violated in the decay, the angular distribution of the proton can be written as 


$$
\frac{d N}{d \Omega}=\frac{1}{4 \Pi}\left(1+a_{\Lambda} \bar{P}_{\Lambda} \cdot \hat{p}\right)
$$

where $\bar{p}$ is the proton's momentum direction in the $\Lambda$ rest frame and the angles $\theta$ and $\phi$ are measured with respect to the $\Lambda$ polarization vector $\overline{\mathrm{P}}_{\Lambda}$. Written in component form

$$
\frac{d N}{d \cos \theta_{i}}=\frac{1}{2}\left(1+a_{\Lambda} P_{\Lambda}^{l} \cos \theta_{l}\right)
$$

where $i=x, y, z$ are axes in the $\Lambda$ rest frame parallel to the spectrometer axes. A plot of the number of protons versus $\cos \theta_{i}$ should give a straight line with slope $a_{\Lambda} P_{\Lambda}{ }^{1 / 2}$. In practice, because of imperfect spectrometer acceptance and reconstruction inefficiencies such a fit is not possible. One can solve the acceptance problem by reversing the sign of the production angle of the hyperons. One has then for + and production angles

$$
\frac{d N^{ \pm}}{d \cos \theta_{i}}=\varepsilon\left(\cos \theta_{i}\right) \frac{1}{2}\left(1 \pm a_{\Lambda} P_{\Lambda}^{i} \cos \theta_{i}\right)
$$

where the sign of the polarization changes with production angle while the acceptance hopefully remains unchanged. The asymmetry

$$
A_{i}=\frac{N_{+}-N_{-}}{N_{+}+N_{-}}=a_{\Lambda} P_{\Lambda}^{i} \cos \theta_{\imath}
$$

is independent of the acceptance and a plot of $A_{i}$ versus $\cos \theta_{i}$ will give a straight line with slope $a_{\Lambda} P_{\Lambda}$.

In reality systematic biases can still exist which will not cancel with the reversal of production angle. For example, the acceptance of the spectrometer may be somewhat different for positive and negative production angles. A direct measure of both the biases and polarization can be made using a hybrid Monte Carlo technique in which Monte Carlo events are generated using the real data to provide the result independent variables. A description of the hybrid Monte Carlo may be found elsewhere 10 . Note however both methods of determining the polarization, asymmetry and hybrid Monte Carlo, give nearly identical results.

\section{$\Xi^{-}$S AND $\Omega^{-}$S FROM PROTONS}

In the first part of $E 756, \Xi^{- \text {'s }}$ and $\Omega^{- \text {'s }}$ were produced directly from $800 \mathrm{GeV}$ protons in Fermilab's Proton Center beamline. The primary proton beam was incident on a $1 / 4 \lambda$ Be target at vertical production angles of $\pm 2.5 \mathrm{mr}$. The Be target was located just outside the hyperon magnet which contained a curved channel. The hyperon magnet and channel served as a dump for the unscattered proton beam, as a momentum selecting and collimating element, and to precess the spin of polarized hyperons. 


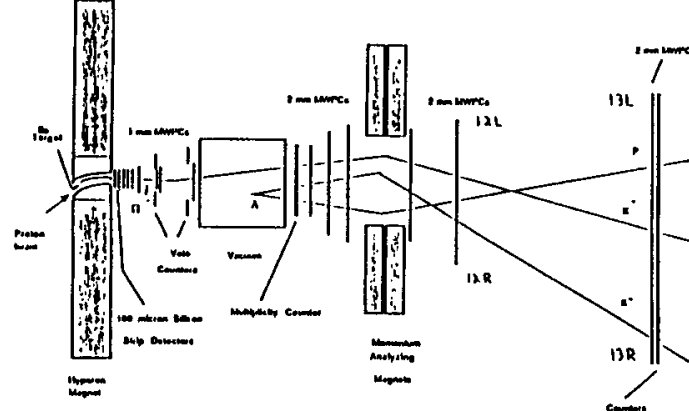

FIG. 2. Plan view of the E756 spectrometer.
Charged particles next entered the spectrometer shown in Figure 2. Differences between this apparatus and a similar setup described previously 11,12 include a set of - 100 micron pitch SSD's, a set of $1 \mathrm{~mm}$ MWPC's, and a dE/dx multiplicity counter. The $\Xi^{-}$ trigger required the coincidence

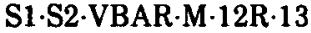

L where a multiplicity requirement of $2 \leq M \leq 4$ and a MWPC requirement of right

half of $\mathrm{C} 12$ and left half of $\mathrm{C} 13$ ensured the correct decay topology. The decays of interest were $\Omega^{-} \rightarrow \Lambda \mathrm{K}^{-}$and $\Xi^{-} \rightarrow \Lambda \mathrm{n}^{-}$where subsequently $\Lambda \rightarrow p \pi^{-}$.

The typical beam intensity for this part of the experiment was $4 \times 10^{10}$ protons per 20 second spill. The production angle was reversed after every pair of data tapes to lessen systematic errors and data was taken at several hyperon magnet field settings. Roughly $5000 \Xi^{-}$triggers were written to tape per spill yielding about 300 $\Xi^{- \text {s }}$ and $4 \Omega^{-}$s per spill. A total of 71 million $\Xi^{-}$triggers written to 200 data tapes will yield 10 million $\Xi^{-1}$ s and 0.1 million $\Omega^{-}$s.

The event reconstruction and selection of $\Xi^{-1} s$ and $\Omega^{- \text {'s }} \mathrm{s}$ was similar to that used in previous experiments 11,13 . The mass plots for $47000 \Omega^{-3} s$ and $75000 \Xi^{-1} s$ are shown in Figures $3 a$ and $b$. The width of the $\Omega^{-}$mass plot is only a few $\mathrm{MeV}$ and the tails are quite small indicating little background.
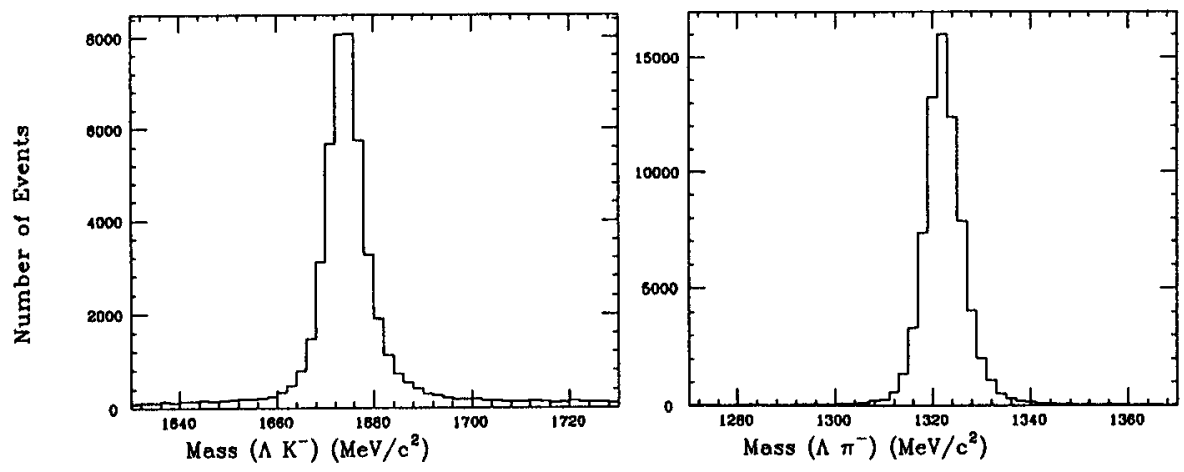

FIG. 3. Invariant mass for a) $\Lambda \mathrm{K}^{-}$and b) $\Lambda \mathrm{\Pi}^{-}$. 
In Figure 4 the polarization of $\Xi^{-9} \mathrm{~s}$ and $\Omega^{-3} \mathrm{~s}$ inclusively produced by protons is presented as a function of transverse momentum. These results are preliminary and

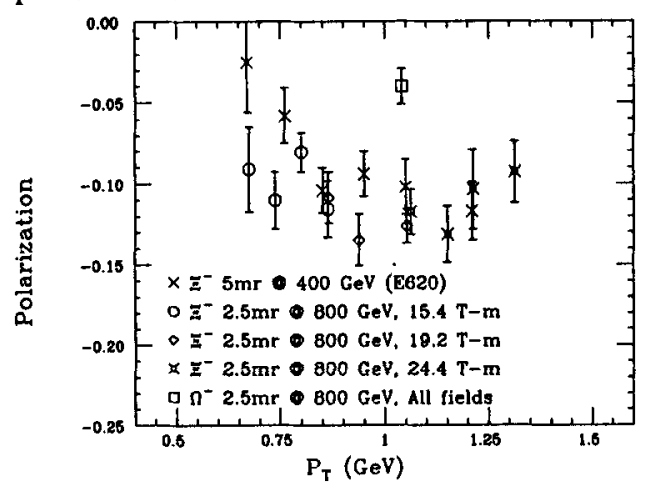

FIG. 4. The $\Xi^{-}$and $\Omega^{-}$polarization for $2.5 \mathrm{mr}$ production by protons. correspond to roughly $10 \%$ of the total $\Xi^{-}$sample and $60 \%$ of the $\Omega^{-}$ sample. Also shown are the results from our previous experiment 11 (E620) at $400 \mathrm{GeV}$. The $\Xi$ polarizations are from several $\int \mathrm{Bdl}$ values of the hyperon magnet while the $\Omega^{-}$polarization is averaged over all fields. For $P_{\mathbf{T}}$ $>.75 \mathrm{GeV}$ the $\Xi^{-}$polarization is slightly larger than $10 \%$ and in good agreement with the E620 result.

That the $\Omega^{-}$s appear to be polarized to a few percent is deceiving since the polarization is found by squaring the $\mathrm{x}$ and $\mathrm{z}$ components. Thus components at different $\int B d l$ having opposite signs will both contribute to a positive result. In Figure 5 , the components of the $\Omega^{-}$polarization in

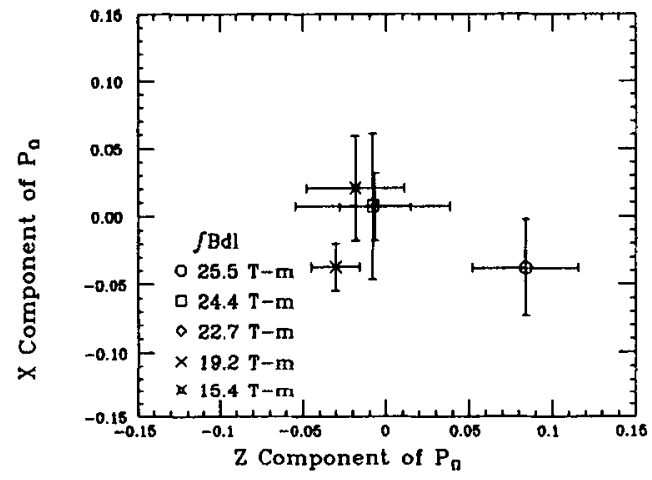

FIG. 5. The $x$ and $z$ components of the $\Omega$ polarization for $2.5 \mathrm{mr}$ production by protons. the $x$ and $z$ directions are shown for the different $\int \mathrm{Bdl}$ values. From this plot we conclude that the polarization of $\Omega^{-}$'s from protons is very small if not zero. Because of the lack of $\Omega^{-}$polarization the $\Omega^{-}$ magnetic moment cannot be measured using $\Omega^{- \text {'s }}$ produced by protons.

Using the hybrid Monte Carlo, one can directly measure the bias in the $\Xi^{-}$polarization measurement. The biases in the $x$ and $\mathrm{z}$ directions are found to be < $2.5 \%$ and do not possess any momentum dependence. The absence of biases is an indication that the data quality is high. by

For $\Xi^{-3}$, the precession angle at a given hyperon magnet field integral is given

$$
\Phi_{n e t}=13.00\left(\frac{g}{2}-1\right) \int B d l
$$

Thus g/ 2 can be extracted and used to determine the magnetic moment via

$$
\mu\left(\Xi^{-}\right)=-.710 \frac{g}{2}
$$


The precession angle at any one $\int \mathrm{Bdl}$ is ambiguous to $\pm \mathrm{nn}$. By measuring $\phi$ at different values of $\int B d l$ and fitting $\phi$ versus $\int B d l$ to a line the ambiguities can be removed, the incorrect values of $n$ giving large $X^{2}$ for the fit line.

In Figure 6 the precession angle for $\Xi^{-1} \mathrm{~s}$ is plotted as a

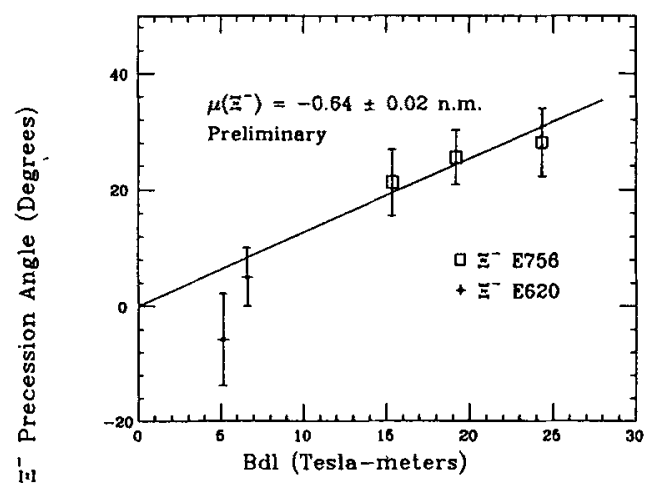
function of hyperon magnet field integral. Results from our previous experiment 14 (E620) are shown also. The value of $(\mathrm{g} / 2-1)$ was determined by fitting a line through the three E756 data points and constrained to 0 at 0 . This gives as our preliminary result for the $\Xi^{-}$magnetic moment $\mu\left(\Xi^{\sim}\right)=-0.64 \pm .02$ n.m. When the $\Xi^{-}$sample is broken into momentum bins, a momentum independent result for the moment is found. The new E756 value is in good agreement with the E620 result of $-0.69 \pm .04 \mathrm{n} . \mathrm{m}$. and a

FIG. 6. The $\Xi^{-}$precession angle versus hyperon magnet $\int B d I$. preliminary result from $\mathrm{E} 715$ reported at this conference 15 of $-0.66 \pm .04$ n.m.

\section{$\Xi^{-\prime}$ S AND $\Omega^{-\prime}$ S FROM A POLARIZED NEUTRAL HYPERON BEAM}

Because inclusively produced $\Omega^{\text {-'s }}$ from protons are unpolarized or polarized very little, alternate schemes for producing a polarized $\Omega^{-}$sample were devised. E756 had two plans for producing polarized $\Omega^{-}$s, the first relying on quark recombination and the other using spin transfer. In the first method, a neutral beam would be produced at $0 \mathrm{mr}$ production angle. Omegas and $\Xi^{-}$s would subsequently be produced at some nonzero production angle by appropriate targetting of this neutral beam. Since the neutral beam would contain $\Xi^{0 \prime}$ s and $\Lambda^{\prime}$ s, the $\Omega^{-}$s produced by it would hopefully be polarized via the same quark recombination mechanism that produces polarized $\Lambda$ 's and $\Xi^{\prime \prime}$ s from protons at $P_{T}$ of about $1 \mathrm{GeV}$.

The second scheme was to initially produce a neutral beam at some production angle. Such a neutral beam would contain polarized $\Xi^{\prime \prime}$ 's, $\Lambda$ 's, and possibly neutrons. This polarized neutral hyperon beam would next be targetted at $0 \mathrm{mr}$ to produce $\Omega^{- \text {'s }}$ and $\Xi^{-1}$ which would hopefully be polarized via spin transfer. It was calculated that the $\Omega^{-}$'s would be produced mainly by $\Xi^{0 \text { "s }}$ with some smaller contribution (roughly $50 \%$ less) from $\Lambda$ 's Because of the configuration of the beamline, it was easier for E756 to implement the spin transfer idea.

For this part of $\mathrm{E} 756$, the $800 \mathrm{GeV}$ proton beam was incident at $\pm 2 \mathrm{mr}$ vertical production angles on a $1 \mathrm{C}$ Cu target. The secondary beam entered a $1.8 \mathrm{~T} 6 \mathrm{~m}$ dipole magnet containing a neutral particle channel. This magnetic channel served to sweep away charged particles and to collimate the neutral beam. The magnetic field was parallel to the parity allowed direction of $\Xi^{\circ}$ and $\Lambda$ polarization so no spin precession occurred. The polarized neutral beam was then incident at $0 \mathrm{mr}$ on another $1 \mathrm{du}$ 
target just outside of the charged hyperon magnet described above. The resultant tertiary beam of $\Xi^{-\prime} s$ and $\Omega^{->}$s passed through the hyperon magnet and spectrometer just as from production by protons.

The intensity for this part of the experiment was approximately $6 \times 1011800$ $\mathrm{GeV}$ protons per $20 \mathrm{sec}$ spill. As above the production angle was reversed after every pair of tapes and data was taken at several values of hyperon magnet $\int B d l$, though most of the running was done at the lowest $\int \mathrm{Bdl}$. Roughly $2200 \mathrm{\Xi}^{-}$triggers were

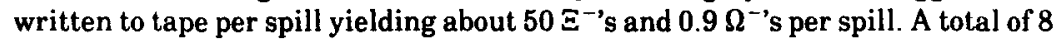
million $\Xi^{-}$triggers written to 140 data tapes will yield 1.5 million $\Xi^{-} \mathrm{s}$ and 22000 $\Omega^{-1}$ s.

The event reconstruction and selection proceeded as for those events produced by protons. Clean mass plots for $\Omega^{- \text {'s }}$ and $\Xi^{- \text {'s }}$ are observed similar to those in Figures $3 a$ and $b$.

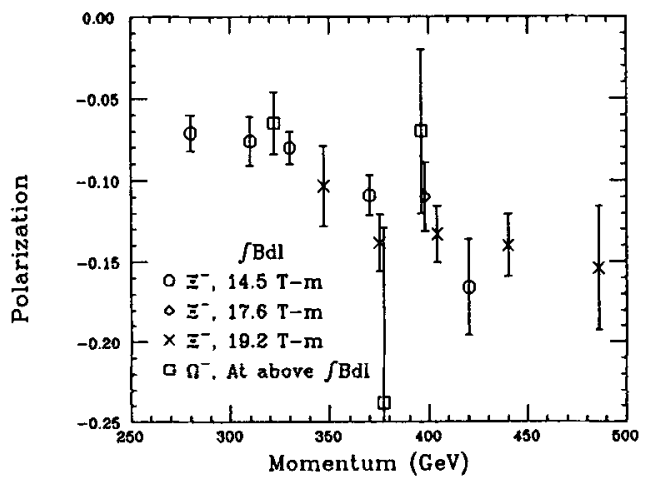

FIG. 7. The $\Xi^{-}$and $\Omega^{-}$polarization for production at $0 \mathrm{mr}$ by a polarized neutral hyperon beam.

The polarization of $\Xi^{-'}$ s and $\Omega^{- \text {'s }}$ from a polarized neutral hyperon beam is shown in Figure 7 as a function of momentum. These results are preliminary and correspond to roughly $20 \%$ of the $\Xi^{-}$data and $100 \%$ of the $\Omega^{-}$data. The $\Xi^{-}$polarization increases from about $7 \%$ at $300 \mathrm{GeV}$ to about $15 \%$ at $400 \mathrm{GeV}$ and above. Clearly significant polarization transfer to $\Xi^{->}$s occurs. The polarization of $\Omega^{- \text {'s }} \mathrm{s}$ is shown for the same three hyperon magnet $\int \mathrm{Bdl}$. The two highest momentum points have small statistics however there is a clear $\Omega^{-}$polarization of $6.5 \%$ at 325 $\mathrm{GeV}$.

The $\Xi^{-}$magnetic moment was found as above by fitting a line through the precession angle versus $\int B d$ points. The moment for $\Xi^{-}$'s produced from the polarized neutral beam agrees within errors with the moment quoted above for $\Xi^{- \text {'s }}$ produced by protons, as expected. The $\Xi^{-}$moment from this part of E756 is found to be independent of the $\Xi^{-}$momentum also.

In Figure 8, the precession angle versus $\int \mathrm{Bdl}$ for $\Omega^{-}$s is given. For $\Omega^{-\prime}$ s, the precession angle is related to $\int \mathrm{Bdl}$ by

$$
\Phi_{\text {net }}=10.27\left(\frac{g}{2}-1\right) \int B d l
$$

and the magnetic moment is found as

$$
\mu\left(\Xi^{-}\right)=-1.683 \frac{g}{2}
$$

Because of the limited statistics at the higher $\int B d l$ values only the precession angle at $14.5 \mathrm{Tm}$ was used in the determination of the $\Omega^{-}$magnetic moment. There is an ambiguity in the precession angle of \pm nn however angles other than 23.5 degrees 


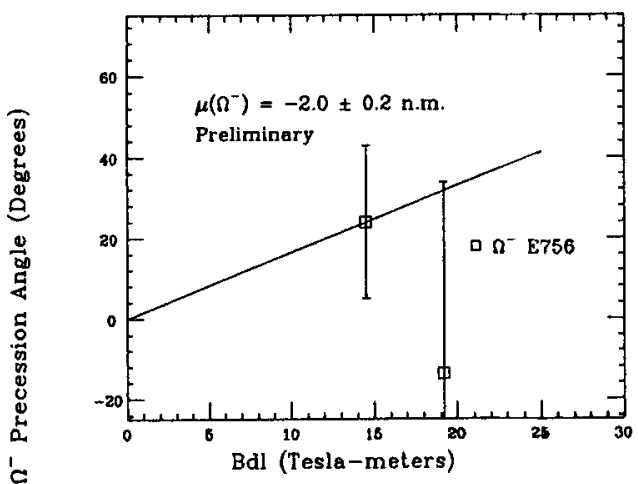

FIG. 8 . The $\Omega^{-}$precession angle versus hyperon magnet $\int \mathrm{Bdl}$. give improbable (positive or large negative) moments. Using the slope of the line determined by this point and $(0,0)$ we find the $\Omega^{-}$ magnetic moment to be $\mu\left(\Omega^{-}\right)=-2.0 \pm 0.2 \mathrm{n} . \mathrm{m}$. (preliminary). This is the first measurement of the $\Omega^{-}$moment. The precession angle at $19.2 \mathrm{Tm}$ (also shown in Figure 8) has a large statistical error but nevertheless gives a magnetic moment that agrees with this value.

To Figure 1 has been added the difference between our measured value for the $\Omega^{-}$moment and various theoretical predictions.

The error bars shown are the $3 \sigma$ error bars of the measurement. In spite of our intuitive feel that the $\Omega^{-}$moment should be easy to calculate there is a dearth of predictions for the $\Omega^{-}$moment. Hopefully this measurement will spur work in that direction. Note also the error bars of the $\Omega^{-}$measurement are much larger than those of any of the other hyperon measurements. They are so large in fact that no theory is ruled out. Although the first measurement of the $\Omega^{-}$moment is rewarding its usefulness is limited. Clearly a high precession measurement of the $\Omega^{-}$moment is needed and has been approved as E800 at Fermilab.

\section{CONCLUSIONS}

We find the polarization of inclusively produced $\Omega^{-}$s by protons to be consistent with zero. A large polarization transfer from a polarized neutral hyperon beam to $\Xi^{-}$'s and $\Omega^{-}$'s is observed. The spin transfer technique produces $\Omega^{- \text {'s }}$ with a polarization of $6.5 \%$ at $325 \mathrm{GeV} / \mathrm{c}$. Our preliminary results for the $\Xi^{-}$and $\Omega^{-}$ magnetic moments measured by spin precession are $\mu\left(\Xi^{-}\right)=-0.64 \pm 0.02 \mathrm{n} . \mathrm{m}$. and $\mu\left(\Omega^{-}\right)=-2.0 \pm 0.2 \mathrm{n} . \mathrm{m}$. An experiment to improve the $\Omega^{-}$magnetic moment precision to $0.03 \mathrm{n} . \mathrm{m}$. has been approved at Fermilab. 


\section{REFERENCES}

1. L. Brekke and J. Rosner, preprint EFI 87-80, (1987).

2. N. Isgur and G. Karl, Phys. Rev. D21, 3175 (1980).

3. H. Georgi and A. Manohar, Phys. Lett. 132B, 183 (1983).

4. R. Verma and M. Khanna, Phys. Lett. 183B, 207 (1987).

5. T. Das and S. Misra, Phys. Lett. 96B, 165 (1980).

6 B. loffe and A. Smilga, Phys. Lett. 133B, 436 (1983).

7. T. Degrand et al,, Phys. Rev D12, 2060 (1975).

8. S. Theberge and A. Thomas, Nucl. Phys. A393, 252 (1983).

9. C. Bernard et al., Phys. Rev. Lett. 49, 1076 (1982).

10. G. Bunce, Nuc. Inst. Meth. 172, $553(1980)$.

11. R. Rameika et al., Phys. Rev. D33, 3172 (1986).

12. L. Deck et al., Phys. Rev. D28, 1 (1983).

13. K.B. Luk et al, Phys. Rev D38, 19 (1988).

14. R. Rameika et al, Phys. Rev. Lett. 52, 581 (1984).

15. C. Newsom, these proceedings. 